\title{
A Novel Algorithm for Impulse Noise Removal and Edge Detection
}

\author{
Akansha Mehrotra \\ Earthquake Engineering \\ IIT Roorkee, Roorkee, India
}

\author{
Krishna Kant Singh \\ Earthquake Engineering \\ IIT Roorkee, Roorkee, India
}

\author{
M.J.Nigam \\ E\&C Department \\ Kirat Pal \\ Earthquake Engineering \\ IIT Roorkee, Roorkee, India
}

\begin{abstract}
Edge detection of images is an important task in computer vision and image processing. Edge detection of noise free images is relatively simpler, but in most practical cases the images are degraded by noise. To find the edges from noisy images is a challenging task. This paper proposes a novel edge detection algorithm for images corrupted with noise. The algorithm finds the edges by eliminating the noise from the image so that the correct edges are determined. For making the image noise free the algorithm calculates closeness parameters, based on this parameter the noisy pixel is replaced by the most appropriate value. The edges of the noise free image are determined using morphological operators erosion and dilation. The proposed algorithm uses a combination of these operators to find the edges. This algorithm uses two different types of structuring elements so that all the edges of the image are determined efficiently.
\end{abstract}

\section{KEYWORDS}

Impulse noise , morphological operators ,edge detection.

\section{INTRODUCTION}

Edges in images provide low-level cues, which can be utilized in higher level processes, such as object detection, recognition, and classification, as well as motion detection, image matching, and tracking [1,2]. Edges and textures in an image are typical examples of high-frequency information. High-pass filters remove low-frequency image information and therefore enhance high-frequency information such as edges. Many approaches to image interpretation are based on edges, since analysis based on edge detection is insensitive to change in the overall illumination level. Edge detection highlights image contrast. Detecting contrast, which is difference in intensity, can emphasize the boundaries of features within an image, since this is where image contrast occurs. This is how human vision can perceive the perimeter of an object, since the object is of different intensity to its surroundings. Edge detection is an important preprocessing step for any image processing application, object recognition and the follow-up analysis. However, the traditional edge detection method (Sobel operator, Prewitt operator, Laplacian operator, etc.) exists widespread paradox between detection accuracy and anti-noise performance[3], recently, edge detection of this problem in mathematical morphology has achieved better results. Mathematical morphology offers a set theoretic approach for image analysis [4,5].The morphological techniques are becoming more and more popular in variety of image processing areas such as image enhancement, object recognition, granulometry, image segmentation, image representation and coding etc.[6]. The main morphological edge detection methods are morphological residual edge detection, top-hat method, ASF method [7], multi-scale method [8] and multi-grade method [9]. Salt-and-pepper impulse noise is one commonly encountered noise type during image and video communication [10].Salt and Pepper noise can be removed by Progressive Switching Median (PSM) filter [11], Extremum Median (EM) filter [12], Adaptive Median (AM) filter [13] and Weighted Median (WM) filter [14] and some other methods like those in $[15,16,21,22]$. Edge detection of noisy images is one of the challenging task in the field of image processing ,the literature survey shows that a number of algorithms have been proposed for this like [17] uses a separate wavelet based noise reduction block for preprocessing before edge detection. The morphological methods to denoise and find edges are proposed in $[18,19]$ but they find edges in a single direction only based on its structuring element, this problem is resolved in [20] which uses eight structuring elements but it is time consuming and increases computational load.

\section{PROPOSED WORK}

In the proposed work a novel edge detection algorithm for noisy images is proposed. The algorithm works in two phases first it makes the image noise free and subsequently finds edges. For finding the edges regions where abrupt changes in grey level occur are to be identified.

PHASE 1: Identification of salt pepper noise: A sliding window $\mathrm{W}_{\mathrm{M}}$ of size $(\mathrm{M}+\Delta) \times(\mathrm{M}+\Delta)$, where $\mathrm{M}=2 \mathrm{~L}+1$ centered at $x_{i j}(\Delta=0$ initially and $M=3)$ is used for detection of pixels as noise free and noise candidates. $\mathrm{S}$ is the set of noise free pixels and $\mathrm{NC}$ is the set containing noise candidate pixels,

$x_{i j} \in \begin{cases}S & \text { if } \min \left[W_{M}\right]<x_{i j}<\max \left[W_{M}\right] \\ N C \quad \text { if } \quad x_{i j}=\min \left[W_{M}\right] \text { or } x_{i j}=\max \left[W_{M}\right]\end{cases}$

Step 1: For the window $\mathrm{W}_{\mathrm{M}}$, the mean of the values of the window is calculated. While calculating the mean only those values are considered whose value lies between $\min \left[W_{M}\right]$ and $\max \left[W_{M}\right]$.

Thus, the mean will be calculated as

$\delta=\frac{1}{n} \sum_{i=0}^{M+\Delta-1} \sum_{j=0}^{M+\Delta-1} P_{i j}$

where $\min \left[W_{M}\right]<\mathrm{P}_{\mathrm{ij}}<\max \left[W_{M}\right]$ and $\mathrm{n}=$ number of pixels whose value lies between $\min \left[W_{M}\right]$ and $\max \left[W_{M}\right]$ 
Step 2: Check if $\delta=0$, this implies that all pixels in the window have the value $\min \left[W_{M}\right]$ or $\max \left[W_{M}\right]$. Now two cases arise as follows:

Case1:If $\min \left[W_{M}\right]=\max \left[W_{M}\right]$, this implies that the window contains a smooth region with all pixels in the window having the same value. Thus, $x_{i j}$ is noise free and need not be replaced

Case2: If $\min \left[W_{M}\right] \neq \max \left[W_{M}\right]$, this implies that the window contains a cluster of noisy pixels. Thus, in this case the window size is increased by 2 (since window size should be an odd number). So, $\Delta=\Delta+2$ using this new value of $\Delta$, mean is calculated again as in step 1, this mean is known as adaptive mean.

Step 3: The next step is to find the most appropriate value which will replace the noisy pixel. For this the closeness parameter of all the pixels in the window $\mathrm{W}_{\mathrm{M}}$, whose value lies between $\min \left[W_{M}\right]$ and $\max \left[W_{M}\right]$ is calculated as follows,

$\sigma_{c}\left(P_{i j}\right)=\left(\delta-P_{i j}\right)$

where $\mathrm{i}=0,1 \ldots(\mathrm{M}+\Delta-1), \mathrm{j}=0,1 \ldots(\mathrm{M}+\Delta-1)$

and $\min \left[W_{M}\right]<\mathrm{P}_{\mathrm{ij}}<\max \left[W_{M}\right]$

The $\sigma_{c}$ form a set $\sigma$ as

$\sigma=\left\{\sigma_{c}: c=0,1 \ldots n\right\}$

where $\mathrm{n}=$ number of pixels whose value lies between $\min \left[W_{M}\right]$ and $\max \left[W_{M}\right]$ The pixel which has the minimum value of $\sigma_{\mathrm{c}}$ will be used to replace the noisy pixel, thus

$\mathrm{R}=P_{i j}$

where $\sigma_{c}\left(P_{i j}\right)=\min \{\sigma\}$

Thus,

$$
x_{i j}=R
$$

Step 4: The above steps are repeated for all the pixels in the set NC.

\section{PHASE 2: Edge Detection}

Once the noise free image denoted by I is obtained it can be used to detect edges. Edges can be detected using the morphological operators like erosion and dilation. Subtracting the eroded image from the original image gives the inner edges of an image while subtracting the original image from the dilated image gives the outer edges of the image. Thus, to find the edges

$G_{\text {in }}=I-(I \ominus B)$

$G_{\text {out }}=(I \oplus B)-I$

$G_{R}=(I \oplus B)-(I \ominus B)$

But, the limitation with this technique is that it finds edges depending on the structuring element. If a single structuring element is used then some edges remain undetected. Thus, in the proposed work two structuring elements are used one for edges in vertical direction and other in horizontal direction as shown in figure 1 (a) and (b).

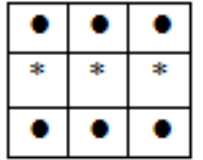

(a)

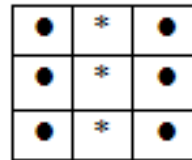

(b)
Figure1 (a) Horizontal structuring element $B_{h}$ (b) Vertical structuring element $B_{v}$

In figure 1, '*' denotes a value and ' $\bullet$ ' denotes a 0 . Using the structuring elements $B_{h}$ and $B_{v}$ horizontal and vertical edges can be found as

$G_{R h}=\left(I \oplus B_{h}\right)-\left(I \ominus B_{h}\right)$

and

$G_{R v}=\left(I \oplus B_{v}\right)-\left(I \ominus B_{v}\right)$

The final edges are a combination of $G_{R h}$ and $G_{R v}$, thus

$G_{F}=\sqrt{G_{R h}^{2}+G_{R v}^{2}}$

Thus, $G_{F}$ contains the final edges of image $I$.

\section{EXPERIMENTAL RESULTS}

In order to study the performance of the proposed algorithm, images have been contaminated with salt pepper noise. The results were compared with several existing methods in both visual image quality and the accuracy with which edges were detected for noisy images. Standard Lena grey image was taken as the test image. Noise of different densities was added ranging from $30 \%$ to $90 \%$. First the noise removal capability of the proposed algorithm(PA) was compared with some of the well known noise removal filters like Standard median $(\mathrm{SM})$, the adaptive median (AM) filter as AM filter shows relatively better performance for noise suppression than other SM filter based methods. Figure 2 shows the original test image LENA. Figure, 3,4 and 5 show the filtering results after adding the salt-and-pepper noises with the noise ratio of $30 \%$ ,60\% and 90\% respectively. For the subjective comparison it is seen that the restoration results using the proposed method are significantly better than the ones by other methods. For the low density noise the suppression result in Figure 3(d) seems almost the same with the original uncorrupted image Figure 2. When the noise ratio reaches as high as $90 \%$, the promising restoration result can be still achieved by using the proposed algorithm, much better than other two filters. Thus, it is seen that the proposed algorithm gives better denoising results than the other methods. The edge detection capabilities of the proposed algorithm (PA) were compared with Sobel operator (SO) and canny edge detector (CD) with. Figure 6,7 and 8 show the result of edge detection of the LENA image of figure 2.It can be seen in figure $6(\mathrm{~b})$ and (c) that with $5 \%$ noise sobel operator and canny edge detector detects noise also as edges while it can be seen that the proposed algorithm finds accurate edges as seen in figure 6(c). With the noise percentage increases to $30 \%$ and $60 \%$ with Sobel and canny edge detector the edges cannot be identified at all while the proposed algorithm works well even with such high noise. The results were measured quantatively using PSNR (Peak Signal to noise Ratio) which is the ratio between the maximum possible power of a signal and the power of corrupting noise that affects the fidelity of its representation. Because many signals have a very wide dynamic range, PSNR is usually expressed in terms of the logarithmic decibel scale. 


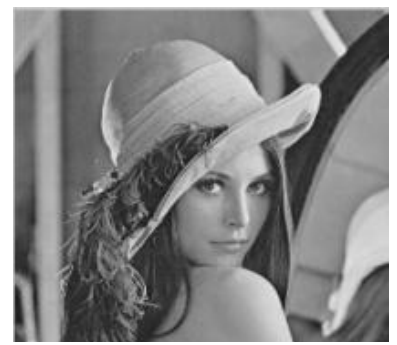

Figure 2 Standard test image LENA

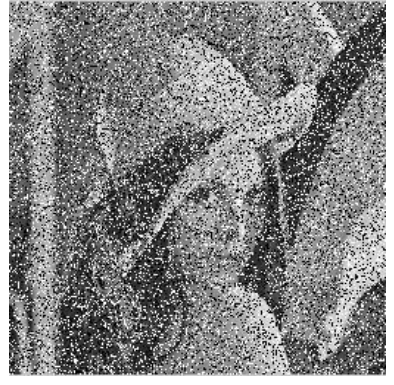

(a)

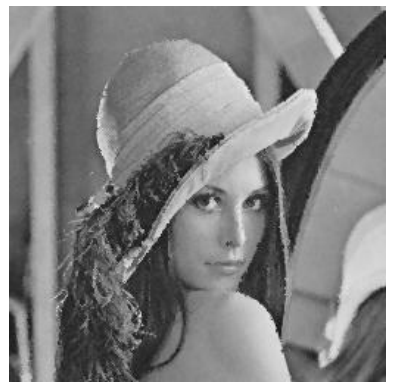

(c)

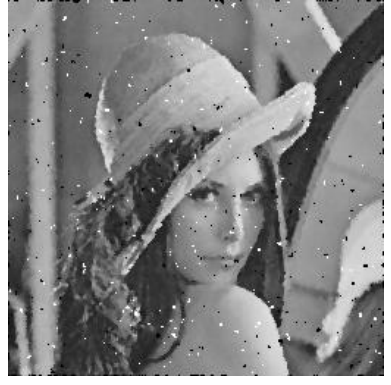

(b)

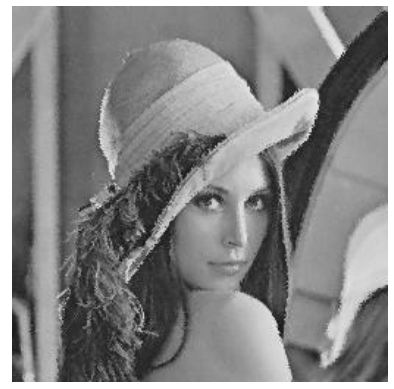

(d)

Figure 3 Restored LENA images using different filters (a) image corrupted with $30 \%$ salt pepper noise (b) SM (c) AM (d) PA

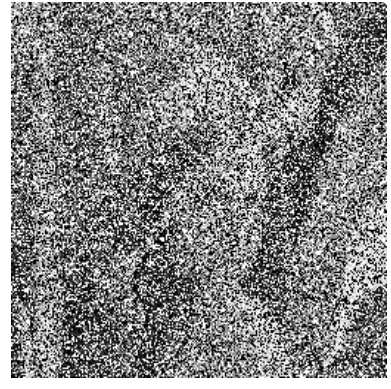

(a)

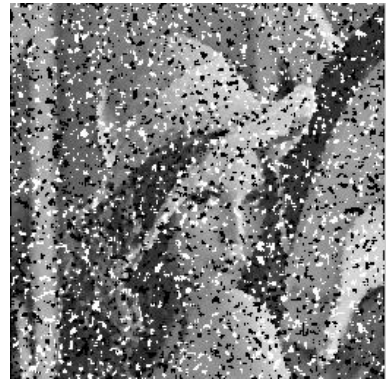

(b)

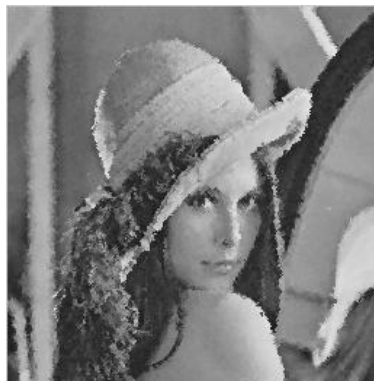

(c)

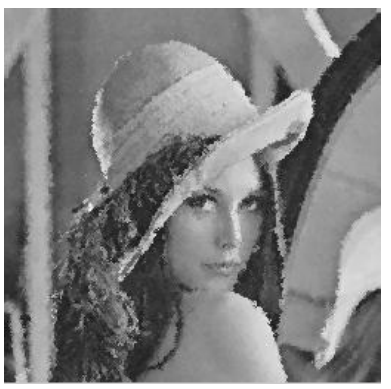

(d)
Figure 4 Restored LENA images using different filters (a) image corrupted with $60 \%$ salt pepper noise (b) SM (c) AM (d) PA

PSNR is a measure of the quality of the reconstruction; a higher PSNR would normally indicate that the reconstruction is of higher quality. It is most easily defined via the mean squared error (MSE) which for two $m \times n$ images $I$ and $K$ where one of the images is considered a noisy approximation of the other is defined as:

$M S E=\frac{1}{m n} \sum_{i=0}^{m-1} \sum_{j=0}^{n-1}[I(i, j)-K(i, j)]^{2}$

The PSNR is defined as:

$$
\begin{aligned}
& P S N R=10 . \log _{10}\left(\frac{M A X_{I}^{2}}{M S E}\right) \\
& =20 . \log _{10}\left(\frac{M A X_{I}}{\sqrt{M S E}}\right)
\end{aligned}
$$

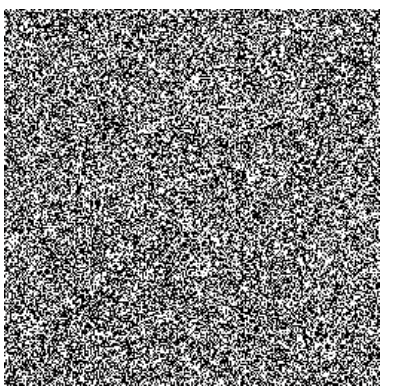

(a)

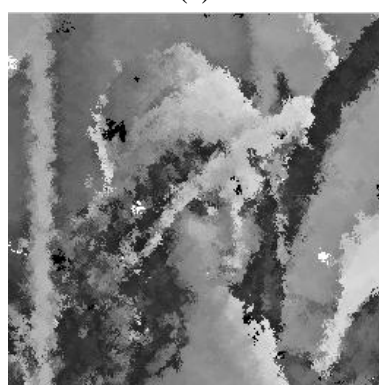

(c)

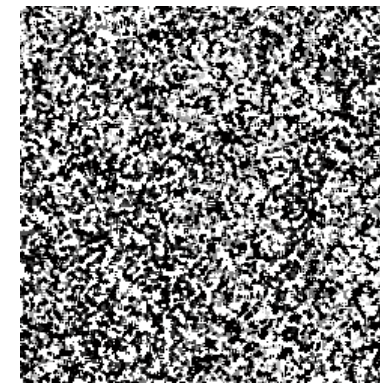

(b)

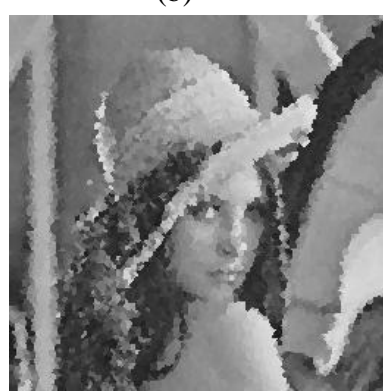

(d)
Figure 5 Restored LENA images using different filters (a) image corrupted with $90 \%$ salt pepper noise (b) SM (c) AM (d) PA 
Here, $M A X_{I}$ is the maximum possible pixel value of the image.Table1 shows the PSNR values of three filters for different noise densities. Figure 9 shows the quantitative comparisons based on PSNR for the different filters.

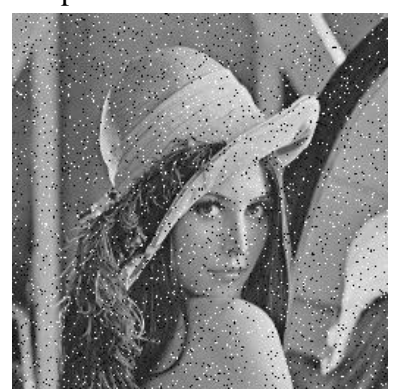

(a)

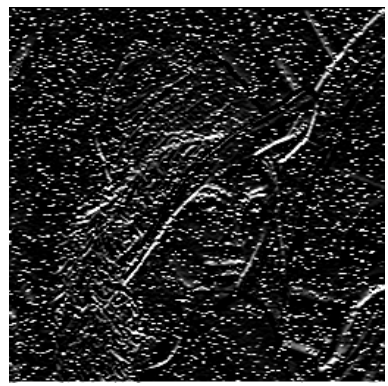

(c)

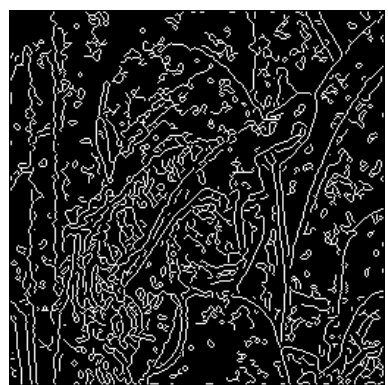

(b)

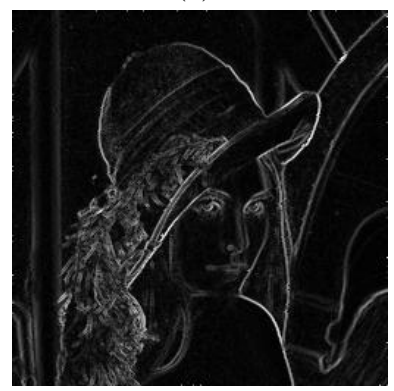

(d)
Figure 6 Edge detection using different filters (a) image corrupted with $5 \%$ salt pepper noise (b) CD (c) SO (d) PA

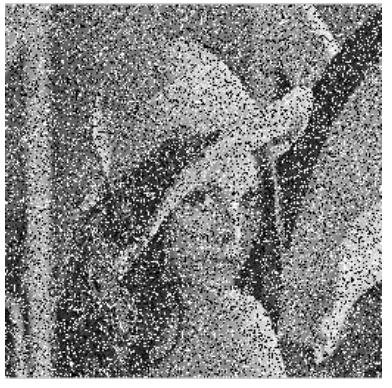

(a)

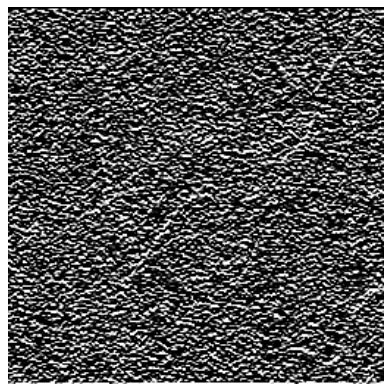

(c)

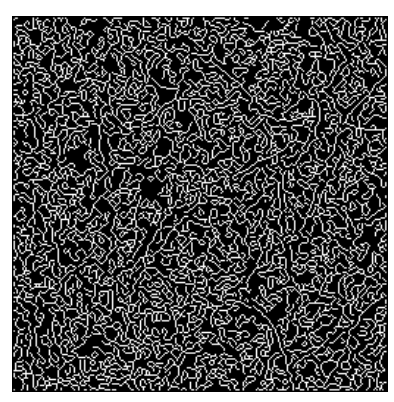

(b)

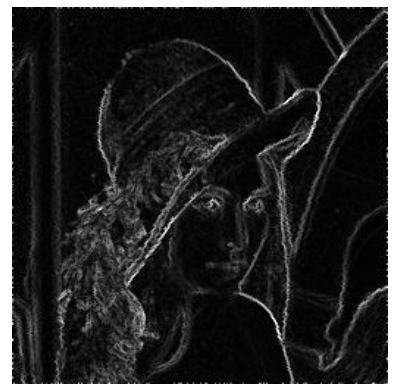

(d)

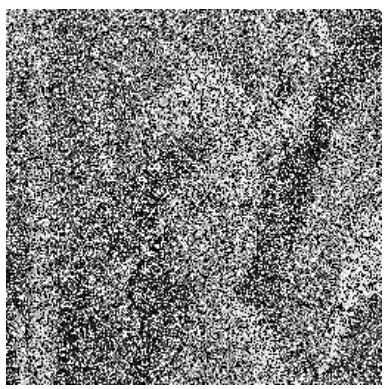

(a)

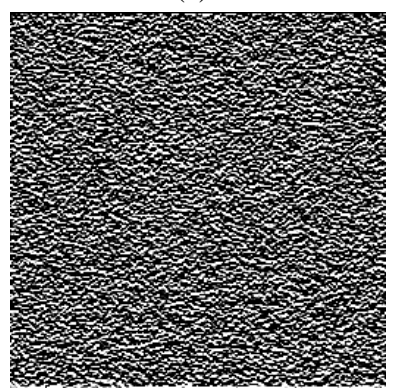

(c)

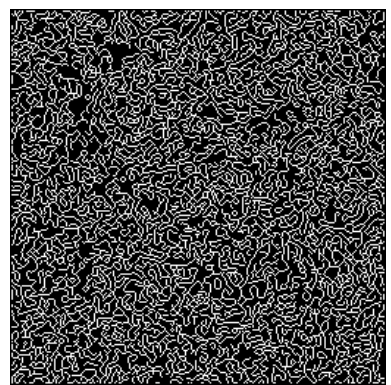

(b)

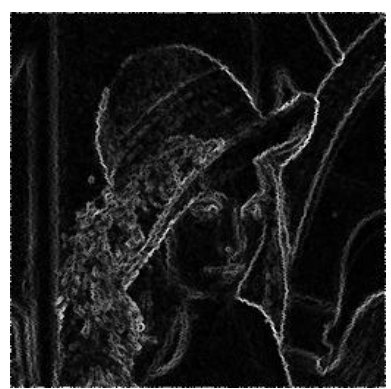

(d)
Figure 8 Edge detection using different filters (a) image corrupted with $60 \%$ salt pepper noise (b) CD (c) SO (d)

PA

\section{CONCLUSION}

A novel algorithm for edge detection of noisy images is proposed. The algorithm first makes the image noise free with the help of a adaptive mean and closeness parameters. The noisy pixels are replaced by a value which is closest to the adaptive mean. The edges are then detected with the help of morphological operators erosion and dilation. Two different structuring elements are used so that all the edges of the image are detected efficiently. Since the proposed method employs a good identification scheme for noise pixels, it shows better performance for the noise removal and also edge detection as compared to other methods. The peak signal to noise ratio also shows improvement as compared to other methods.

TABLE 1

PSNR OF DIFFERENT FILTERS FOR DIFFERENT NOISE DENSITIES

\begin{tabular}{|l|l|l|l|}
\hline $\begin{array}{l}\text { Noise } \\
\text { level }\end{array}$ & $\begin{array}{l}\text { Standard } \\
\text { Median }\end{array}$ & $\begin{array}{l}\text { Adaptive } \\
\text { Median }\end{array}$ & $\begin{array}{l}\text { Proposed } \\
\text { Algorithm }\end{array}$ \\
\hline $30 \%$ & 22.1454 & 28.8738 & 28.8784 \\
\hline $60 \%$ & 12.0530 & 24.3270 & 24.4751 \\
\hline $80 \%$ & 8.1378 & 21.3660 & 22.7459 \\
\hline $90 \%$ & 6.6231 & 18.3907 & 21.3305 \\
\hline $95 \%$ & 5.9764 & 14.3164 & 19.9958 \\
\hline
\end{tabular}

Figure 7 Edge detection using different filters (a) image corrupted with $30 \%$ salt pepper noise (b) CD (c) SO (d) 


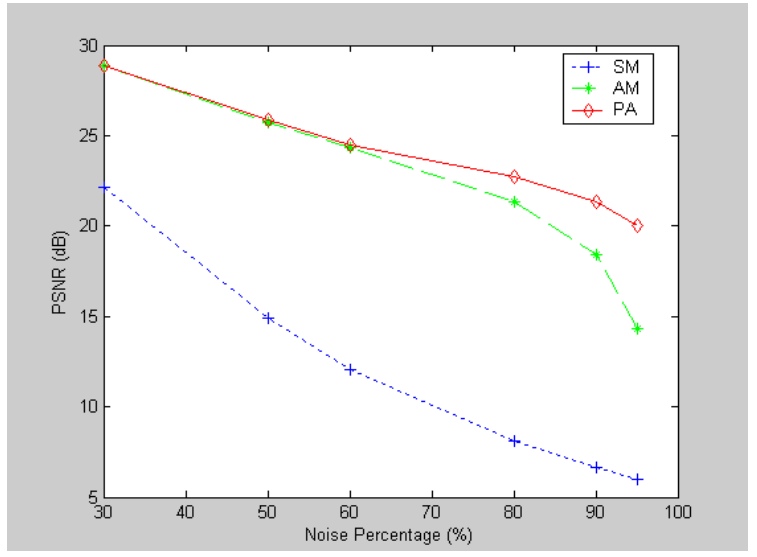

Fig.9 Results in PSNR for different filters

\section{REFERENCES}

[1] H. Bay, V. Ferraris, and L. Van Gool. Wide-baseline stereo matching with line segments. IEEE Conf. on Comp. Vis. And Patt. Rec., 1:329 -336, 2005.

[2] K. Mikolajczyk, A. Zisserman, and C. Schmid. Shape recognition with edge-based features. British Mach. Vis. Conf., 2:779 - 788, 2003.

[3] Zhao CH, Zhang Q. "The edge detection of medical image based on mathematics morphological filtering operators." Information Technology, vol.11, no.1, 2002, pp.49-50

[4] J. Serra, Image Analysis and Mathematical Morphology, Vol. 1 and 2, Acad. Press, NY, 1983 and 1988.

[5] C. Giardina and E. Dougherty, Morphological Methods in Image and Signal Processing, Prentice Hall, Englewood Cliffs, 1988 .

[6] P. Maragos and R. Schafer, "Morphological Systems for Multidimensional Signal Processing" Proc. IEEE,Vol. 78,No. 4,690-710,1990.

[7] X. Song, and Y.Neuvo, "Robust edge detection based on morphological filters", Pattern Recognition Lett., Vol. 14, 1993, pp. 889-894.

[8] B.Chanda, K.K.Malay, and Y.V.Padmaja, "A multiscale morphologic edge detection", Pattern Recognition, Vol. 31, 1998, pp. 1469-1478.

[9]M.Y.Jiang, and D.F.Yuan, "A multi-grade mean morphologic edge detection", 6th International Conference on Signal Processing, Beijing, China, 2002, pp.1079-1082.

[10] Raymond H. Chan, Chung-Wa Ho, and Mila Nikolova,"Salt-and-pepper noise removal by mediantype noise detectors and detail-preserving regularization," IEEE Transactions on Image Processing, vol. 14, no. 10, pp. 1479-1485, Oct. 2005.
[11] Z. Wang, D. Zhang, "Progressive switching median filter for the removal of impulse noise from highly corrupted images," IEEE Transactions on Circuits and Systems, II : Analog and Digital SignalProcessing, vol. 46, pp. 78-80, Jan. 1999

[12] C. Xing, S. Wang, H. Deng, and Y. Luo. "A new filtering algorithmbased on extremum and median value," Journal of Image and Graphics,vol. 6, pp. 533-536, Jun. 2001.

[13] H. Hwang, A. Haddad, "Adaptive median filters: New algorithms and results," IEEE Transactions on Image Processing, vol. 4, pp. 499-502,Apr. 1995.

[14] D. Brownrigg, "The weighted median filter," Communication Association Computer Machine, vol. 27, pp. 807-818, Aug. 1984.

[15] Changhong Wang, Taoyi Chen and Zhenshen $\mathrm{Qu}$ "A Novel Improved Median Filter for Salt-and-Pepper Noise from Highly Corrupted Images" in proc. Systems and Control in Aeronautics and Astronautics (ISSCAA), 2010 p.718 - 722

[16] Dagao Duan, Qian Mo,Yueliang Wan and Zhongming Han "A Detail Preserving Filter for Impulse Noise Removal" in Proc.ICCASM,2010,paper V2-265

[17] Feng-ying Cui , Li-jun Zou and Bei Song, "Edge Feature Extraction Based on digital Image processing techniques,'Proc. IEEE Int'l conference Automation and logistics, Qingdao,China September 2008

[18] Chunhua Li,Kun He and Jiliu Zhou , "Edge Detection of Image on the local feature, "Second international symposium on Intelligent technology Application 2008.

[19] Zhao Yu-qian, Gui Wei-hua, Chen Zhen-cheng, Tang Jing-tian, Li Ling-yun, "Medical images based on Mathematical Morphology, "Proc. IEEE Engineering in Medicine and Biology 27th Annual conference, Shanghai , China , September 1-4 , 2005.

[20] Yuqian Zhao, Weihua Gui and Zhencheng Chen "Edge Detection Based on Multi structure Elements Morphology,"Proc. IEEE Intelligent Control and Automation, June 21-23,2006,Dalian, China.

[21] Krishna Kant Singh, Akansha Mehrotra , Kirat Pal, M.J.Nigam, "A N8(P) Detail Preserving Adaptive Filter For Impulse Noise Removal” ,Proceedings IEEE, 2011 International Conference on Image Information Processing (ICIIP 2011).

[22.] Krishna Kant Singh, Akansha Mehrotra, M.J.Nigam, Kirat Pal, "A Novel Edge Preserving Filter For Impulse Noise Removal”, Proceedings IEEE, IMPACT 2011. 